\title{
Fluorine-18-Fluorodeoxyglucose-Avid Thyroid Incidentalomas on Positron Emission Tomography/Computed Tomography in Patients with Non-Thyroid Malignancy: Incidence and Possible Nature
}

\author{
DALIA K. SEROUR, M.D.* and AYMAN M.A. OSMAN, M.D., M.R.C.S.** \\ The Departments of Diagnostic \& Interventional Radiology* and General Surgery**, Faculty of Medicine, \\ Cairo University, Egypt
}

\begin{abstract}
Background: Incidental thyroid lesions carry the risk of being malignant. Hence, they present a potential challenge for clinicians that necessitates careful management.

Aim of Study: The aim of this retrospective cohort study was to assess the thyroid incidentalomas detected on fluorine18-Fluorodeoxyglucose Positron Emission Tomography/ Computed Tomography ( ${ }^{18}$ F-FDG PET/CT), as well as to determine a maximal standardized uptake value (SUVmax) cutoff that could potentially help in the diagnostic process.

Patients and Methods: Medical records of 1526 patients who performed F-FDG PET/CT as part of the diagnostic work up for non-thyroid malignancy were retrospectively reviewed. Patients with thyroid incidentalomas who fulfilled the eligibility criteria were enrolled into the study. The SUVmax of the lesions and the results of histopathological examination were recorded. Receiver-Operating Characteristic (ROC) curve analysis was carried out.
\end{abstract}

Results: Thyroid incidentalomas were detected in 112/1526 patients $(7.3 \%)$. Of those, only 46 patients (41\%) fulfilled the eligibilty criteria. Histopathological examinations [FNAC $(n=46)$, surgery $(n=19)]$ revealed that $11 / 46$ lesions $(23.9 \%)$ were malignant, whereas $35 / 46$ lesions $(76.1 \%)$ were benign. The mean SUVmax of malignant lesions $(7.05 \pm 1.51)$ was significantly higher than that of benign lesions (3.18 \pm 1.74$)$; $p=0.001$. An SUVmax cutoff value of 5.5 was the most accurate for discriminating between benign and malignant incidentalomas, with $90.91 \%$ sensitivity and $82.86 \%$ specificity; $p=0.001$.

Conclusion: The incidence of ${ }^{18}$ F-FDG-avid thyroid incidentalomas is relatively high among patients who undergo $\mathrm{PET} / \mathrm{CT}$ as part of the diagnostic work up for non-thyroid malignancy. The SUVmax values are higher in malignant incidentalomas, yet with a noticeable overlap in the values between benign and malignant lesions. An SUVmax cutoff value of 5.5 can potentially be utilized to predict the nature of incidental thyroid lesions.

Correspondence to: Dr. Dalia K. Serour, E-Mail: daliakhaled80@yahoo.com
Key Words: Incidental thyroid lesions - Thyroid incidentalomas - Positron emission tomography/computed tomography - Maximal standardized uptake value Cutoff value.

\section{Introduction}

INCIDENTAL thyroid lesions (thyroid incidentalomas) are asymptomatic thyroid lesions that are accidentally discovered while investigating another unrelated condition. Thyroid incidentalomas represent a frequent radiological/clinical finding, occurring in up to $5 \%$ of the population. The risk of those lesions is generally higher in females, and increases with age, reaching up to $30-40 \%$ among patients over the age of 50 . Incidental thyroid lesions carry the risk of being malignant. Hence,

\footnotetext{
List of abbreviations:

US : Ultrasound.

CT : Computed Tomography.

MRI : Magnetic Resonance Imaging.

SUVmax : Maximal Standardized Uptake Value.

FDG : Fluorodeoxyglucose.

PET/CT : Positron Emission Tomography/Computed Tomography.

3D : Three-Dimentional.

PET : Positron Emission Tomography.

PSF : Point-Spread-Function.

TOF : Time-of-Flight.

FNAC : Fine-Needle Aspiration Cytology.

ROC : Receiver-Operating Characteristic.

SPSS : Statistical Package for Social Sciences.

SD : Standard Deviation.

NOS : Not Otherwise Specified.

MIP : Maximum Intensity Projection.

F-FDG : Fluorine-18-Fluorodeoxyglucose Positron Emission PET Tomography.

F-FDG : Fluorine-18-Fluorodeoxyglucose Positron Emission PET/CT Tomography/Computed Tomography.
} 
they present a potential challenge for clinicians that necessitates careful management $[\mathbf{1 , 2}]$.

The recent generation of Ultrasound (US) machines, which provides better resolution compared to previous generations, has demonstrated an increased depiction rate of incidental thyroid lesions, reaching up to $67 \%$, a rate which is more or less equivalent to that provided by autopsy [3]. In addition, several prospective studies have revealed a $70-83 \%$ depiction rate of small incidental thyroid nodules that are smaller than $10 \mathrm{~mm}$ on US scanning [4]. Meanwhile, a depiction rate of $15 \%$ has been reported with the use of Computed Tomography (CT) or Magnetic Resonance Imaging (MRI) [5,6], compared to a rate of $1-2 \%$ with the use of fluorine18-fluorodeoxyglucose positron emission tomography ( ${ }^{8}$ F-FDG PET) $[\mathbf{7}, 8]$.

The aim of this retrospective cohort study was to assess the incidental thyroid lesions detected on ${ }^{18}$ F-FDG positron emission tomography/computed tomography ( ${ }^{18}$ F-FDG PET/CT) in terms of FDG uptake patterns, maximal standardized uptake values (SUVmax), and incidence of malignancy; as well as to determine an SUVmax cutoff value that could potentially help in the diagnostic process.

\section{Patients and Methods}

The medical records of 1526 patients who per-

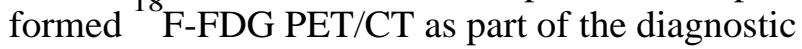
work up for non-thyroid malignancy between December 2019 and June 2020 in our institution were retrospectively reviewed. Patients in whom ${ }^{18}$ F-FDG PET/CT revealed a thyroid incidentaloma were enrolled into the study only if they fulfilled the following stringent eligibility criteria:

\section{- Inclusion criteria:}

Documented area(s) of abnormal Fluorodeoxyglucose (FDG) uptake in the thyroid gland; no history of thyroid cancer or previous thyroid surgery; as well as documented results of histopathological examination of the F-FDG-avid thyroid incidentaloma.

\section{- Exclusion criteria:}

History of thyroid cancer, previous thyroid surgery, and/or unavailable histopathological data.

The study protocol was approved by the institutional Ethical Committee.

\section{Imaging technique:}

- All examinations were performed on a dedicated PET/CT scanner (Siemens, CT 128; Medical Solutions, Knoxville, USA).
- All patients had to fast for almost six hours before the examination.

- In supine position; the examination was performed with the patient's arms resting over the head, and via helical mode, the acquisition of images was carried out from the head down to the mid-thigh level.

- In a three-dimentional (3D) mode; a Positron Emission Tomography (PET) scan was performed following a low-dose Computed Tomography (CT) scan.

- Images were acquired using sequential bed positions in an overlapping pattern, with a scan duration of 3 minutes per bed position.

- The image reconstruction method incorporated Point-Spread-Function (PSF), along with Timeof-Flight (TOF). Finally, via 3-mm slice thickness, the given projection data was reconstructed.

- To obtain multiplanar reformatted CT images, a special work station was used.

Data analysis and interpretation of the PET/CT images:

All ${ }^{18}$ F-FDG PET/CT images were interpreted by two radiologists, one of whom interpreted all the images of the study patients, whereas the second radiologist varied from one patient to the other. Each of the reporting radiologists had an experience of 5 years-at least-in PET/CT imaging.

Thyroid affection was classified into focal and diffuse FDG uptake patterns. The focal uptake pattern was defined as a localized form of FDG uptake, whereas the diffuse uptake pattern was defined as FDG uptake entangling the whole thyroid gland.

A cubical volume of interest, $30-\mathrm{mm}$ in size, was placed over the suspicious ${ }^{\text {F-FDG-avid }}$ thyroid lesion to obtain the maximal standardized uptake value (SUVmax). In the case of diffuse thyroid gland affection, the area of maximal FDG uptake was visually detected and its SUVmax was calculated. In the case of multiple thyroid nodules, the SUVmax was used to quantify the FDG uptake in the nodule with the highest uptake. The calculated SUVmax was correlated with background activity where the liver was used as a reference value. However, in cases with a diseased liver; the mediastinal blood pool was used instead.

Finally, all the study patients underwent diagnostic thyroid Fine-Needle Aspiration Cytology (FNAC). The FNAC results were categorized into 4 groups: Benign, suspicious for malignancy, 
malignant, and inconclusive. If the result was 'malignant', total thyroidectomy was carried out. If it was 'suspicious for malignancy', either total thyroidectomy or thyroid lobectomy was undertaken, depending on the pattern and distribution of FDG uptake. However, if the result was 'benign', thyroid lobectomy was carried out only in patients with focal FDG uptake and a corresponding suspicious solitary thyroid nodule on clinical examination. The definitive diagnosis of the nature of the incidental thyroid lesion(s) was made on the basis of histopathological examination (surgery/FNAC).

Patient socio-demographic data, FDG uptake patterns and maximal standardized uptake values (SUVmax) of the incidental thyroid lesions, as well as results of histopathological examination of those lesions were all recorded. Previous imaging studies and relevant clinical data were also documented.

\section{Statistical analysis:}

Values were expressed in the form of mean \pm standard deviation or number (\%). A $p$-value $<0.05$ was regarded as an indicator for significant difference. Receiver-Operating Characteristic (ROC) curve analysis was then used to determine an SUVmax cutoff value that could potentially help to discriminate between benign and malignant thyroid incidentalomas. Data was analyzed via Statistical Package for Social Sciences (SPSS) software.

\section{Results}

Of the 1526 patients who underwent ${ }_{18}^{18}$ F-FDG PET/CT over the 7-month study period, ${ }^{18}$ F-FDGavid thyroid incidentalomas were detected in 112 patients, with an incidence (depiction rate) of $7.3 \%$. Of those, only 46 patients (41\%) fulfilled our eligibilty criteria and were enrolled into the study.

The study patients $(n=46)$ ranged in age from 32 to 83 years [mean \pm Standard Deviation (SD), $59.30 \pm 12.07]$. Fourteen patients $(30.4 \%)$ were males, whereas $32(69.6 \%)$ were females; $p=0.008$. The clinical features of the study patients and their primary malignant pathologies were listed in (Tables 1,2).

Table (1) clinical features of the study patients $(n=46)$.

Table (2) primary malignant pathologies in the study patients $(n=46)$.

The FNAC results of the ${ }^{18}$ F-FDG-avid incidental thyroid lesions were 'malignant' in 9 cases (19.6\%), 'suspicious for malignancy' in 8 cases
(17.4\%), and 'benign' in 29 cases (63\%). Based on the F-FDG PET/CT findings and FNAC results, thyroid surgery was carried out in a total of 19 cases $(41.3 \%)$ [total thyroidectomy $(n=12)$, thyroid lobectomy $(n=7)]$. Histopathological examinations (surgery/FNAC) revealed that 11 out of the 46 incidental thyroid lesions $(23.9 \%)$ were malignant [papillary thyroid carcinoma $(n=5)$, thyroid lymphoma $(n=3)$, thyroid metastases $(n=2)$, follicular thyroid carcinoma $(n=1)]$; whereas 35/46 lesions (76.1\%) were benign [benign, Not Otherwise Specified (NOS) $(\mathrm{n}=22)$; thyroiditis $(\mathrm{n}=7)$; multinodular goiter $(\mathrm{n}=6)]$ (Table 3$)$.

Table (1): Clinical features of the study patients $(n=46)$.

\begin{tabular}{|c|c|c|}
\hline Variable & Number & Percent \\
\hline Age (years, mean \pm SD) & $59.30 \pm 12.07$ & \\
\hline \multicolumn{3}{|l|}{ Gender: } \\
\hline Female & 32 & $69.6 \%$ \\
\hline Male & 14 & $30.45 \%$ \\
\hline \multicolumn{3}{|l|}{ Pattern of FDG uptake: } \\
\hline Diffuse & 14 & $30.4 \%$ \\
\hline Focal & 32 & $69.6 \%$ \\
\hline \multicolumn{3}{|l|}{ Thyroid lobe(s) involved: } \\
\hline Left lobe & 17 & $37.0 \%$ \\
\hline Right lobe & 12 & $26.1 \%$ \\
\hline Both lobes & 17 & $37.0 \%$ \\
\hline FNAC & 46 & $100 \%$ \\
\hline Surgery & 19 & $41.3 \%$ \\
\hline \multicolumn{3}{|l|}{$\begin{array}{l}\text { Pathology of incidental } \\
\text { thyroid lesions: }\end{array}$} \\
\hline Benign & 35 & $76.1 \%$ \\
\hline Malignant & 11 & $23.9 \%$ \\
\hline \multicolumn{3}{|c|}{$\begin{array}{l}\text { FDG : Fluorodeoxyglucose. } \\
\text { FNAC: Fine Needle Aspiration Cytology. }\end{array}$} \\
\hline \multicolumn{3}{|c|}{ Table (2): Primary malignant pathologies in the study patients. } \\
\hline Primary malignant pathology & $\begin{array}{l}\text { Number } \\
(\%)\end{array}$ & $\begin{array}{c}\text { Malignant } \\
\text { incidental } \\
\text { thyroid lesions } \\
(\mathrm{n}=11)\end{array}$ \\
\hline Breast cancer & $19(41.3 \%)$ & 4 \\
\hline Lymphoma & $4(8.6 \%)$ & \\
\hline Urinary bladder carcinoma & $1(2.2 \%)$ & \\
\hline Ovarian Cancer & $3(6.5 \%)$ & 1 \\
\hline Pancreatic Cancer & $1(2.2 \%)$ & \\
\hline Colon Cancer & $3(6.5 \%)$ & \\
\hline Renal cell carcinoma & $3(6.5 \%)$ & 2 \\
\hline Primary peritonteal carcinoma & $1(2.2 \%)$ & \\
\hline Hepatocellular carcinoma & $1(2.2 \%)$ & \\
\hline Lung cancer & $1(2.2 \%)$ & 1 \\
\hline Sarcoma & $2(4.3 \%)$ & 1 \\
\hline Prostate cancer & $1(2.2 \%)$ & \\
\hline Endometrial carcinoma & $1(2.2 \%)$ & \\
\hline Esophageal cancer & $1(2.2 \%)$ & \\
\hline Melanoma & $1(2.2 \%)$ & \\
\hline Gastric carcinoma & $3(6.5 \%)$ & 2 \\
\hline
\end{tabular}


Table (3) results of histopathological examination for incidental thyroid lesions in the study patients.

The FDG uptake pattern was diffuse in 14 out of the 46 incidental thyroid lesions $(30.4 \%)$, and focal in 32/46 lesions (69.6\%). In the 'diffuse uptake pattern' group, $12 / 14$ lesions $(85.7 \%)$ were benign [thyroiditis $(n=7)$, benign, NOS $(n=5)]$; whereas only $2 / 14$ lesions $(14.3 \%$ ) were malignant (thyroid lymphomas); $p=0.002$. On the other hand, in the 'focal uptake pattern' group in which 9/32 lesions $(28.1 \%)$ were malignant, the size of the malignant lesions [1.8-2.7cm (mean \pm SD, 1.69 \pm $0.87)]$ was larger than that of the benign lesions [0.9-2.7cm (mean $\pm \mathrm{SD}, 0.93 \pm 0.77)]$, with a statis- tically significant difference between the two given groups $(p=0.002)$.

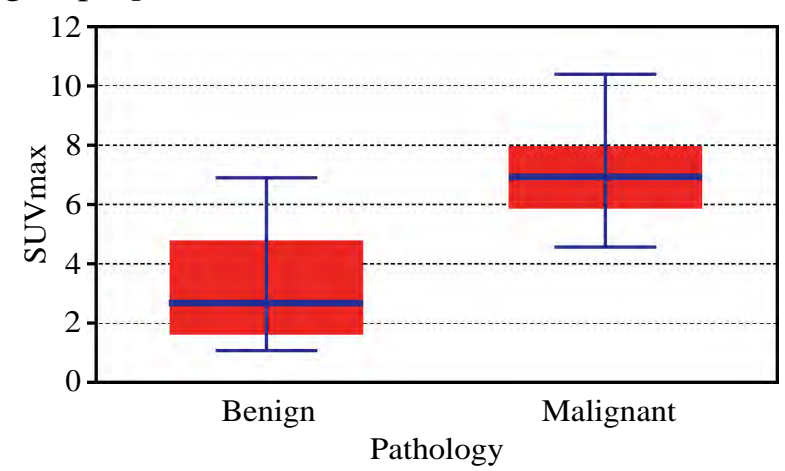

Fig. (1): Boxplot showing the maximal standardized uptake values (SUVmax) of incidental thyroid lesions in the study patients.
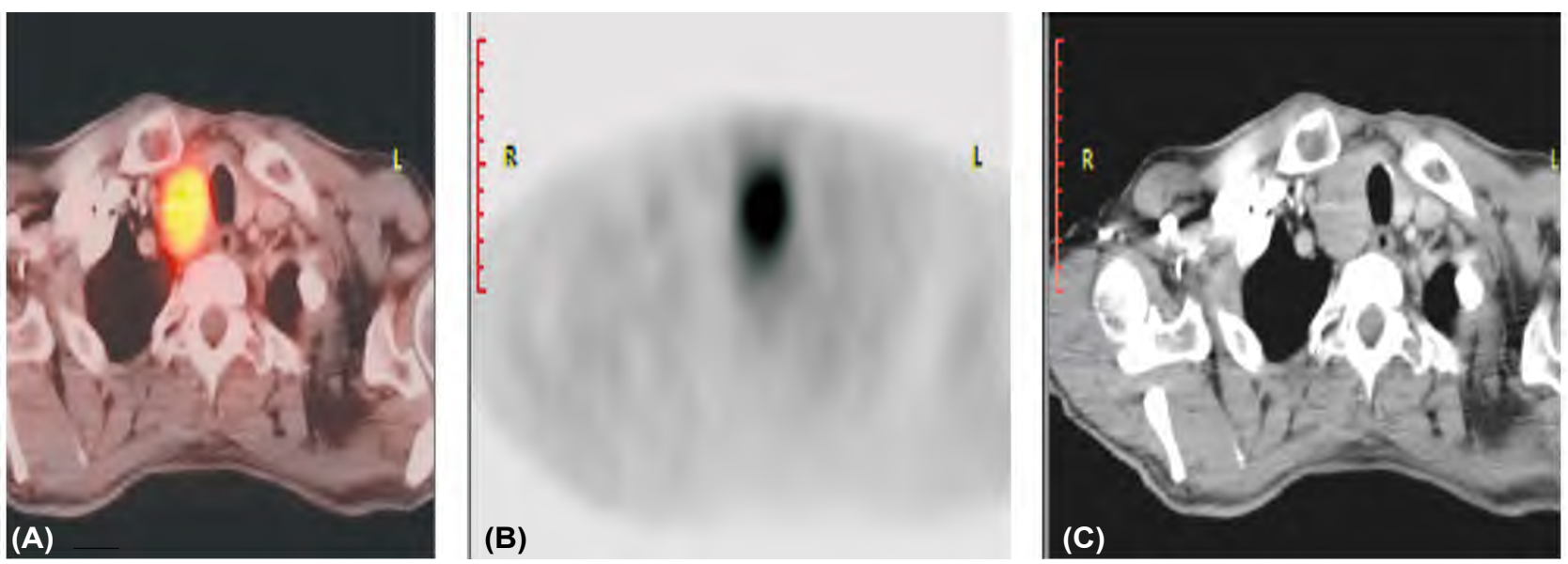

Fig. (2): Positron Emission Tomography/Computed Tomography (PET/CT) in a patient with esophageal cancer showing an ${ }^{18}$ F-FDG-avid thyroid incidentaloma, with the (A) Axial fused, (B) Axial MIP and (C) Axial CT images revealing a right thyroid lobe well-defined hypodense nodule showing heterogeneous contrast uptake associated with intense FDG avidity (SUVmax 5.8). Histopathological examination of the lesion revealed its benign nature.

${ }^{18}$ F-FDG : Fluorine-18-Fluorodeoxyglucose.
MIP : Maximum Intensity Projection.

CT : Computed Tomography.

SUVmax : Maximal Standardized Uptake Value.
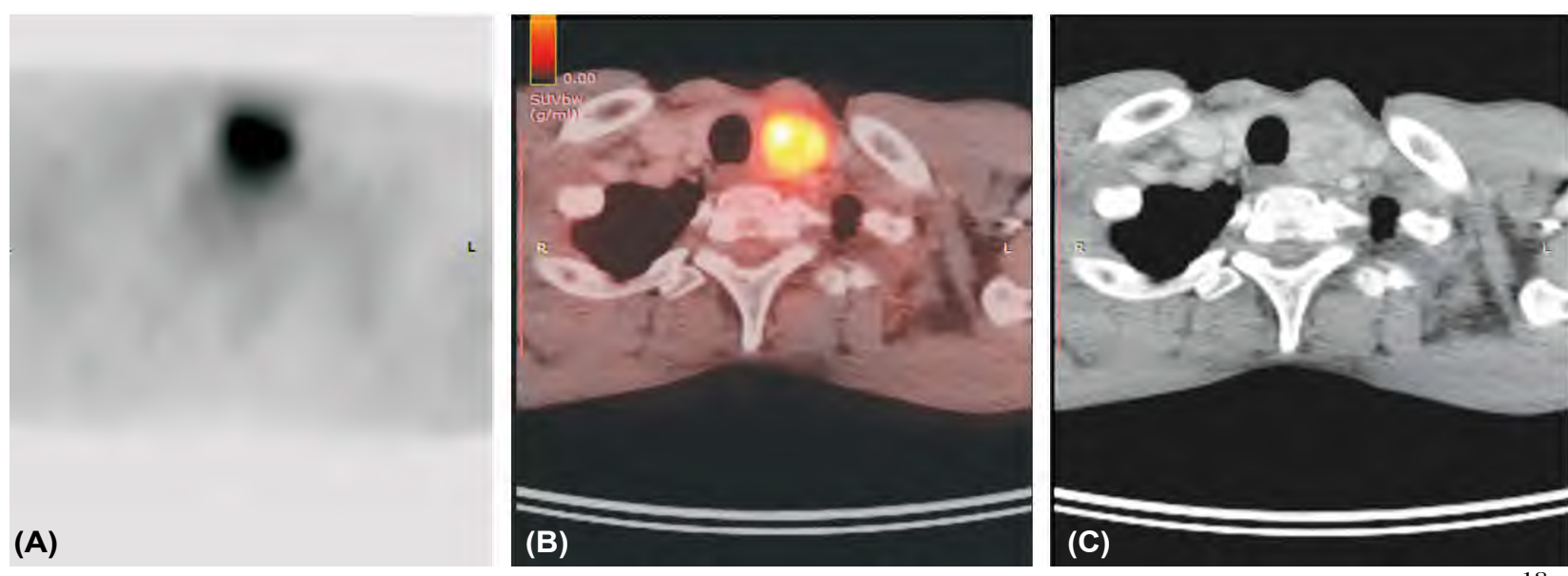

Fig. (3): Positron Emission Tomography/Computed Tomography (PET/CT) in a patient with breast cancer showing an ${ }^{18}$ FFDG-avid thyroid incidentaloma, with the (A) Axial fused, (B) Axial MIP and (C) Axial CT images revealing a left thyroid lobe hypermetabolic calcified nodule (SUVmax $~ 7.8$ ). Histopathological examination of the lesion revealed papillary thyroid carcinoma.

\footnotetext{
${ }^{18}$ F-FDG : Fluorine-18-Fluorodeoxyglucose.

MIP : Maximum Intensity Projection.
} 

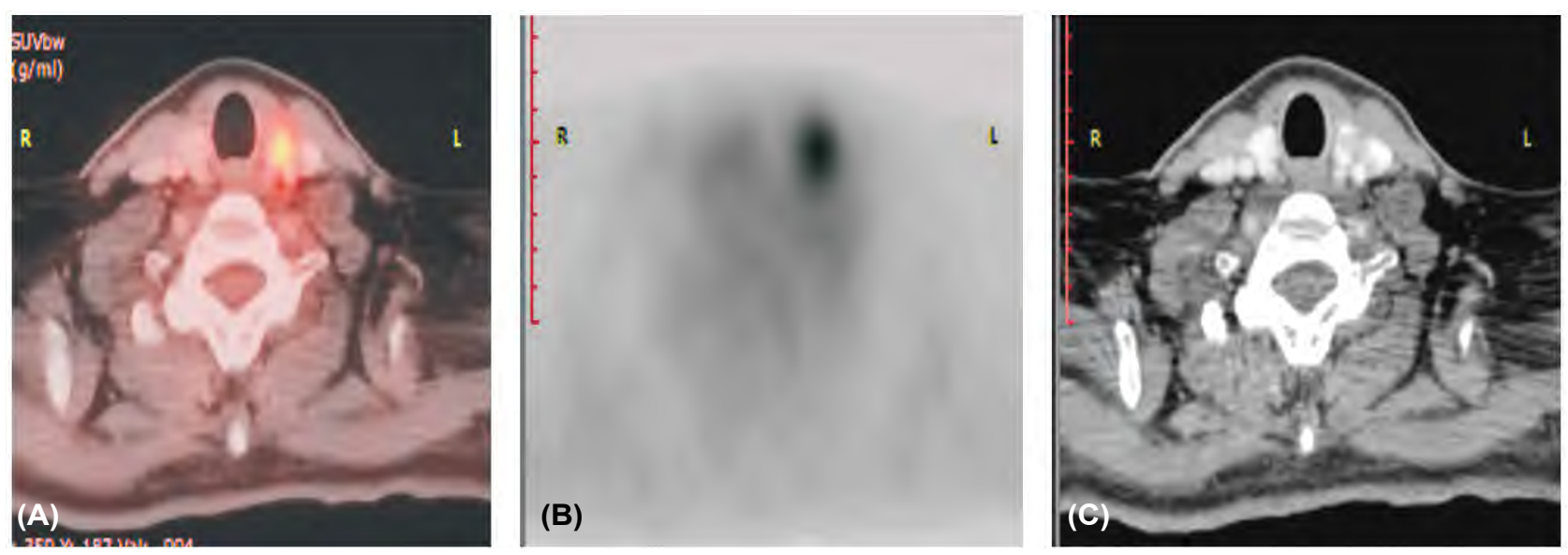

Fig. (4): Positron Emission Tomography/Computed Tomography (PET/CT) in a patient with breast cancer showing an ${ }^{18}$ FFDG-avid thyroid incidentaloma, with the (A) Axial fused, (B) Axial MIP and (C) Axial CT images revealing a left thyroid lobe small hypermetabolic nodule (SUVmax 3.6). Histopathological examination of the lesion revealed its benign nature.

${ }^{18}$ F-FDG : Fluorine-18-Fluorodeoxyglucose.
MIP : Maximum Intensity Projection.
CT : Computed Tomography.

SUVmax : Maximal Standardized Uptake Value.

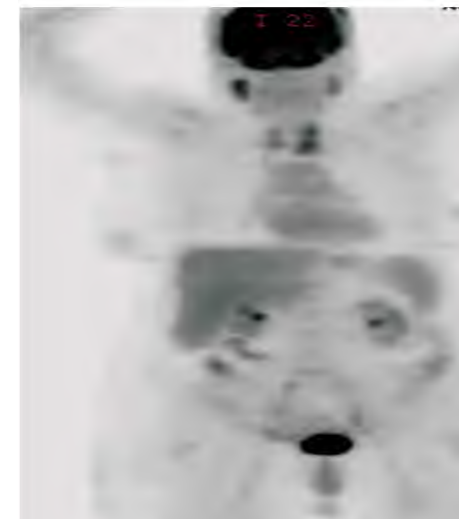

(A)

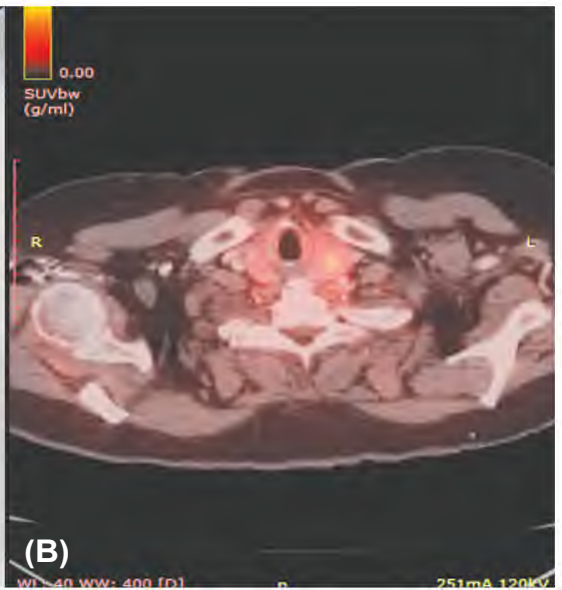

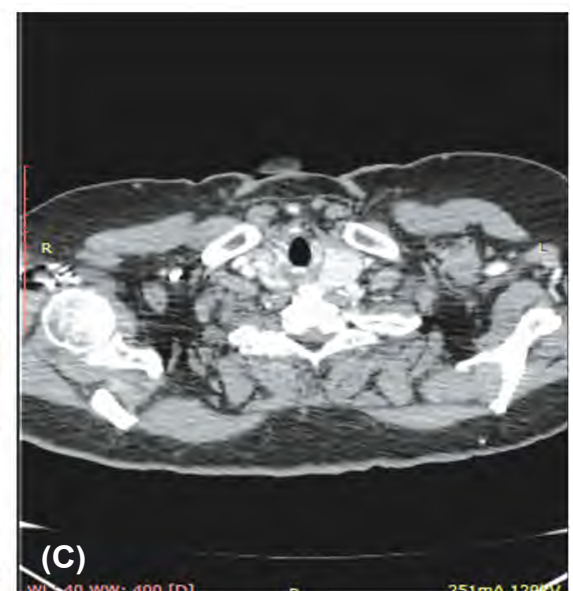

Fig. (5): Positron Emission Tomography/Computed Tomography (PET/CT) in a patient with endometrial carcinoma showing an ${ }^{18}$-FDG-avid thyroid incidentaloma, with the (A) Coronal MIP, (B) Axial fused, and (C) Axial CT images revealing diffuse thyroid uptake, more on the left side (SUVmax 5.9). Histopathological examination of the lesion revealed thyroiditis.

$$
\begin{aligned}
& { }^{18} \text { F-FDG : Fluorine-18-Fluorodeoxyglucose. } \\
& \text { MIP : Maximum Intensity Projection. }
\end{aligned}
$$

In the study patients, the maximal standardized uptake values (SUVmax) of the malignant lesions [4.5-10.3 (mean $\pm \mathrm{SD}, 7.05 \pm 1.51)$ ] were higher than those of the benign lesions [1.2-6.4 (mean \pm $\mathrm{SD}, 3.18 \pm 1.74)]$; and the difference between the two given groups was statistically significant; $p=$ 0.001 Figs. (1-5).

Receiver-Operating Characteristic (ROC) curve analysis showed that the SUVmax cutoff value of 5.5 was the most accurate for discriminating between benign and malignant thyroid incidentalomas (i.e. an SUVmax $>5.5$ was strongly suggestive of malignancy), with an area under the curve of 0.949 , a sensitivity of $90.91 \%$, a specificity of $82.86 \%$,
CT : Computed Tomography.

SUVmax : Maximal Standardized Uptake Value.

a positive predictive value of $62.5 \%$, and a negative predictive value of $96.7 \%$; $p=0.001$ Fig. (6).

Table (3): Results of histopathological examination for incidental thyroid lesions in the study patients.

Biopsy results

Number (\%)

\section{Malignant:}

Papillary thyroid carcinoma

$5(10.9 \%)$

Thyroid lymphoma

$3(6.5 \%)$

Thyroid metastases

$2(4.3 \%)$

Follicular thyroid carcinoma

$1(2.2 \%)$

Benign:

Benign NOS (Non Otherwise Specified) $24(52.2 \%)$

Thyroiditis

$7(15.2 \%)$

Multinodular goiter 


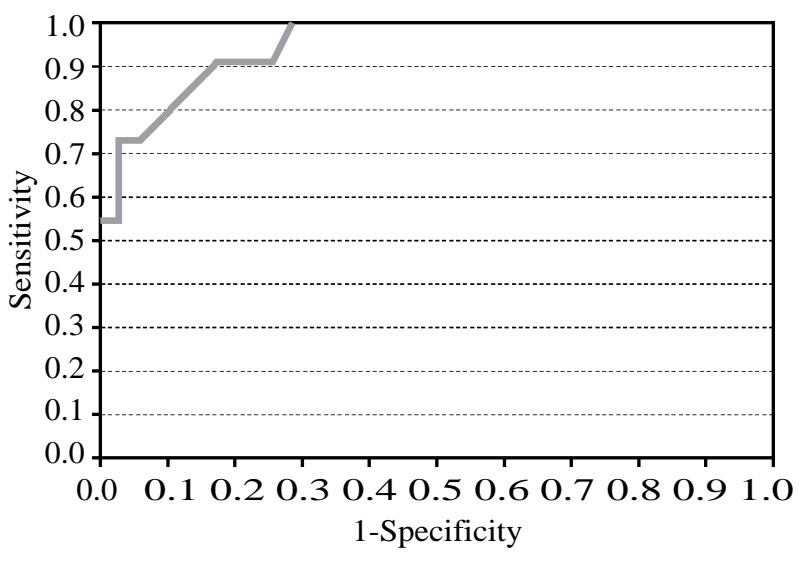

Fig. (6): Receiver-Operating Characteristic (ROC) curve analysis showing that the SUVmax cutoff value of 5.5 was the most accurate for discriminating between benign and malignant thyroid incidentalomas, with an area under the curve of 0.949 , a sensitivity of $90.91 \%$ and a specificity of $82.86 \%$; $p=0.001$.

SUVmax: Maximal Standardized Uptake Value.

\section{Discussion}

Incidental lesions or incidentalomas at different anatomical sites (e.g. thyroid gland, parotid gland, breast, suprarenal glands, gastrointestinal tract, prostate); presenting as areas of increased or ab-

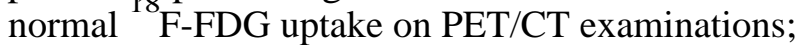
have all been studied throughout the literature. Although the thyroid gland has long been considered as the best example for evaluation of incidentalomas detected on F-FDG PET/CT, the assessment of such thyroid lesions still presents a big source of challenge and controversy $[9,10]$.

With the recent significant increase in the use of PET/CT technology \& the number of PET/CT machines all over the world, and with the emergence of new indications for this examination; there has been an increase in the number of incidental thyroid lesions detected. Thereby, evaluating such lesions and identifying their possible nature, whether malignant or benign, has become crucial [11-14].

Our study revealed a significantly higher incidence of thyroid incidentalomas in females (69.6\%), compared to males $(30.4 \%) ; p=0.008$. This finding is consistent with Choi et al., [11] who reported a female-to-male ratio of 25:19 in patients with incidental thyroid lesions, as well as Bae et al., [13] who also demonstrated an increased incidence of such lesions in females $(11.7 \%$ in females compared to $4.7 \%$ in males), with $p<0.0001$ denoting statistical significance. It also partially agrees with Algin et al., [15] who reported a relatively higher incidence in females, compared to males, yet with no significant statistical difference.
In this study, 112 cases of thyroid incidentalomas, showing diffuse or focal FDG uptake patterns, were detected on ${ }^{18}$ F-FDG PET/CT, with an incidence (depiction rate) of $7.3 \%$, and a $23.9 \%$ incidence of malignancy. These findings agree with Algin et al., [15] who reported incidental thyroid lesions, showing diffuse or focal uptake patterns, in $5.6 \%$ of cases, with a $32.7 \%$ incidence of malignancy. They are also consistent with Bae et al., [13] and Kang et al., [14], who reported an up to 9\% prevalence of incidental thyroid lesions. The higher incidence of thyroid incidentalomas in our study could be largely attributed to the enrollment of patients showing either diffuse or focal FDG uptake patterns in contrary to most of other studies which were primarily concerned about the focal uptake pattern, revealing a lower incidence of thyroid lesions $(1.1-4 \%)$, with a $14-60 \%$ incidence of malignancy $[11,12,16]$.

The thyroid gland diffuse FDG uptake pattern has almost been related to benign causes (e.g. multinodular goiter, thyroiditis) [17]. In our study, in the 'diffuse uptake pattern' group, most of the thyroid incidentalomas were benign [12/14 lesions $(85.7 \%)]$, whereas only $2 / 14$ lesions $(14.3 \%)$ were malignant; and the difference was statistically significant $(p=0.002)$. The incidence of malignancy in the aforementioned group was lower than that observed in the 'focal uptake pattern' group (28.1\%). These findings agree with Bae et al., [13] who reported a $30.9 \%$ incidence of malignancy in lesions showing focal FDG uptake, compared to a $6.4 \%$ incidence in those showing diffuse uptake. They also agree with Kang et al., [14] who reported a $37.2 \%$ incidence of malignancy in lesions showing focal uptake, compared to a $4.7 \%$ incidence in those showing diffuse uptake. However, our findings disagree with Algin et al., [15] who showed no statistically significant difference in incidence between benign and malignant lesions in patients with diffuse FDG uptake. In our study, thyroiditis was the most commonly depicted benign pathology in the 'diffuse uptake pattern' group, whereas thyroid lymphoma was the most commonly depicted malignant pathology in the aforementioned group. This observation is consistent with the results of a recent study [15] which showed that the commonest benign cause for diffuse FDG uptake was thyroiditis, whereas the commonest malignant causes for this uptake pattern were thyroid metastases and lymphoma. Our findings also agree with previous results reported by Basu et al., [18] and Karantanis et al., [19].

Data analysis showed that the mean size of the malignant lesions $(1.69 \pm 0.87 \mathrm{~cm})$ was larger than 
that of the benign lesions $(0.93 \pm 0.77 \mathrm{~cm})$, with a statistically significant difference between the two groups $(p=0.002)$. This agreed with Algin et al., [15] who reported a statistically significant difference between the mean size of malignant lesions $(2.9 \pm 1.6 \mathrm{~cm})$ and that of benign lesions $(2.1 \pm 0.9 \mathrm{~cm})$; $p=0.014$. On the contrary, however, Bae et al., [13] showed no significant difference in size between benign and malignant lesions ( $p=0.289$ ). Our study also revealed that the mean maximal standardized uptake value (SUVmax) of the malignant lesions (7.05 \pm 1.51$)$ was higher than that of the benign lesions (3.18 \pm 1.74$)$, and the difference between the two groups was also statistically significant; $p=0.001$. In addition, ROC curve analysis showed that the SUVmax cutoff value of 5.5 was the most accurate for predicting the nature of thyroid incidentalomas, with $90.91 \%$ sensitivity and $82.86 \%$ specificity. These findings agree with Algin et al., [15] who reported a statistically significant difference between benign and malignant thyroid lesions in terms of their SUVmax $(p<0.001)$, while suggesting a cutoff value of 6 to predict the nature of the lesion. Our results also agree with 3 other studies $[12,14,16]$ which reported statistically significant differences between benign and malignant incidental thyroid lesions in terms of their maximal standardized uptake values, with the values being higher in malignant lesions. In contrast to our study findings, Bogsrud et al., [20] and Kim et al., [21] showed no significant difference in SUVmax between benign and malignant thyroid incidentalomas.

In this study, there was a noticeable overlap in the maximal standardized uptake values (SUVmax) between benign and malignant incidental thyroid lesions. This was one of the main challenges encountered by the reporting radiologists during interpretation of the PET/CT images. Similar patterns of overlap were noted in previous studies $[12,14-16]$. Few theories have been proposed to explain this overlap. The most widely accepted theory was that based on the expression of glucose transporters in thyroid incidentalomas, the pattern of which could explain the difference in the degree of FDG uptake between benign and malignant lesions [22].

\section{Limitations of the study:}

The study limitations included the lack of histopathological data in some cases with thyroid incidentalomas, the retrospective study design, as well as the small sample size. In addition, the possibility of missing a thyroid malignancy in patients with multiple thyroid nodules showing abnormal FDG uptake was another drawback, where only the nodule with the highest FDG uptake was histopathologically evaluated by FNAC.

\section{Conclusion:}

The incidence of ${ }^{18}$ F-FDG-avid thyroid incidentalomas is relatively high among patients who undergo PET/CT as part of the diagnostic work up for non-thyroid malignancy. The maximal standardized uptake values (SUVmax) are higher in malignant incidentalomas, yet with a noticeable overlap in the values between benign and malignant lesions. An SUVmax cutoff value of 5.5 can potentially be utilized to predict the nature of incidental thyroid lesions. Further prospective studies are, however, still needed in order to explore in depth the role of ${ }^{18}$ F-FDG PET/CT in detecting and predicting the nature of those frequently encountered thyroid lesions.

\section{References}

1- HEGEDÜS L., BONNEMA S.J. and BENNEDBAEK F.N.: Management of simple nodular goiter: Current status and future perspectives. Endocr. Rev., 24: 102-32, 2003.

2- MAZZAFERRI E.L.: Management of a solitary thyroid nodule. N. Engl. J. Med., 328: 553-9, 1993.

3- EZZAT S., SARTI D.A., CAIN D.R. and BRAUNSTEIN G.D.: Thyroid incidentalomas. Prevalence by palpation and ultrasonography. Arch. Intern. Med., 154: 1838-40, 1994.

4- BRANDER A.E., VIIKINKOSKI V.P., NICKELS J.I. and KIVISAARI L.M.: Importance of thyroid abnormalities detected at US screening: A 5-year follow-up. Radiology, 215: 801-6, 2000.

5- YOUSERM D.M., HUANG T., LOEVNER L.A. and LANGLOTZ C.P.: Clinical and economic impact of incidental thyroid lesions found with CT and MR. AJNR Am. J. Neuroradiol., 18: 1423-8, 1997.

6- YOON D.Y., CHANG S.K., CHOI C.S., YUN E.J., SEO Y.L., NAM E.S., CHO S.J., RHO Y.S. and AHN H.Y.: The prevalence and significance of incidental thyroid nodules identified on computed tomography. J. Comput. Assist. Tomogr., 32: 810-5, 2008.

7- SOELBERG K.K., BONNEMA S.J., BRIX T.H. and HEGEDUS L.: Risk of malignancy in thyroid incidentalomas detected by ${ }^{1} 8$ F-fluorodeoxyglucose positron emission tomography: A systematic review. Thyroid, 22: 91825, 2012.

8- SHETTY S.K., MAHER M.M., HAHN P.F., HALPERN E.F. and AQUINO S.L.: Significance of incidental thyroid lesions detected on CT: Correlation among CT, sonography, and pathology. AJR Am. J. Roentgenol., 187: 1349$56,2006$.

9- HAN E.J., CHOI W.H., YOO I.R. and CHUNG S.K.: Significance of incidental focal uptake in prostate on $18_{-}$ fluoro-2-deoxyglucose positron emission tomography CT images. Br. J. Radiol., 83: 915-20, 2010.

10- BASU S., HOUSENI M. and ALAVI A.: Significance of incidental fluorodeoxyglucose uptake in the parotid glands 
and its impact on patient management. Nucl. Med. Commun., 29: 367-73, 2008.

11- CHOI J.Y., LEE K.S., KIM H.J., SHIM Y.M., KWON O.J., PARK K., BAEK C.H., CHUNG J.H., LEE K.H. and KIM B.T.: Focal thyroid lesions incidentally identified by integrated F-FDG PET/CT: Clinical significance and improved characterization. J. Nucl. Med., 47: 60915, 2006.

12- COHEN M.S., ARSLAN N., DEHDASHTI F., et al.: Risk of malignancy in thyroid incidentalomas identified by fluorodeoxyglucose-positron emission tomography Surgery, 130 pp. 941-6, 2001.

13- J.S. BAE, B.J. CHAE, W.C. PARK, et al.: Incidental thyroid lesions detected by FDG-PET/CT: Prevalence and risk of thyroid cancer. World J. Surg. Oncol., 7 p. 63, 2009.

14- B.J. KANG, O. J.H., J.H. BAIK, S.L. JUNG, Y.H.PARK and S.K. CHUNG: Incidental thyroid uptake on F- ${ }^{\text {FDG }}$ PET/CT: Correlation with ultrasonography and pathology. Ann. Nucl. Med., 23 pp. 729-37, 2009.

15- ALGIN E.F., UNER A.Y., OZAGUR E.A., GUMUSAY O.Z., KAPUCU O.Z. and OZET,A.H.: The assessment of incidental thyroid lesions on F-fluorodeoxyglucose positron emission tomography/computed tomogrophy: A single centre experience, Journal of Oncological Sciences, 3: 57-61, 2017.

16- CHEN Y.K., DING H.J., CHEN K.T., et al.: Prevalence and risk of cancer of focal thyroid incidentaloma identified by F-fluorodeoxyglucose positron emission tomography for cancer screening in healthy subjects Anticancer Res., 25, pp. 1421-6, 2005.

17- S. KURATA, M. ISHIBASHI, Y. HIROMATSU, et al.: Diffuse and diffuse-plus-focal uptake in the thyroid gland identified by using FDG-PET: Prevalence of thyroid cancer and Hashimoto's thyroiditis. Ann. Nucl. Med., 21 pp. 325-30, 2007.

18- BASU S., LI G., BURAL G. and ALAVI A.: Fluorodeoxyglucose positron emission tomography (FDG-PET) and PET/computed tomography imaging characteristics of thyroid lymphoma and their potential clinical utility Acta Radiol, 50 pp. 201-4, 2009.

19- KARANTANIS D., BOGSRUD T.V., WISEMAN G.A., et al.: Clinical significance of diffusely increased ${ }^{\mathrm{F}}$ FDG uptake in the thyroid gland J. Nucl. Med., 48 pp. 896-901, 2007.

20- BOGSRUD T.V., KARANTANIS D., NATHAN M.A.,

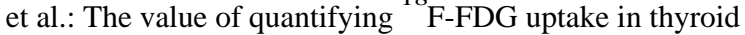
nodules found incidentally on whole-body PET-CT Nucl. Med. Commun., 28, pp. 373-81, 2007.

21- KIM T.Y., KIMW.B., RYU J.S., GONG G., HONG S.J., SHONG Y.K.: $\quad$ F-fluorodeoxyglucose uptake in thyroid from positron emission tomogram (PET) for evaluation in cancer patients: High prevalence of malignancy in thyroid PET incidentaloma, Laryngoscope, 115 pp. 1074$8,2005$.

22- C. MIAN, S. BAROLLO, G. PENNELLI, et al.: Molecular characteristics in papillary thyroid cancers (PTCs) with no 131I uptake Clin Endocrinol (Oxf), 68 pp. 108-16, 2008.

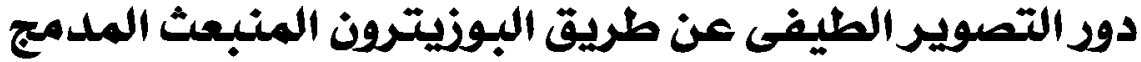

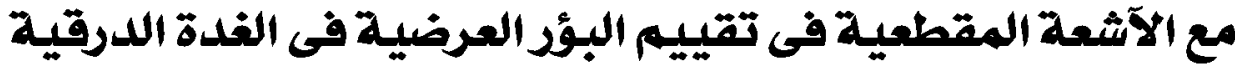

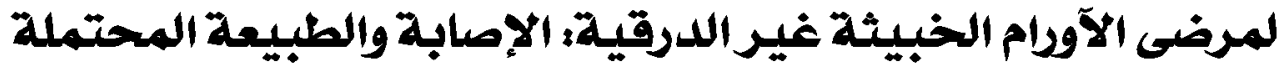

\footnotetext{
البؤد العرضية آو الآودام المكتشفة بالصدفة فى مواقع تشريحية مختلةة مثل الغدة الدرقية، الغدة النكفية، الثدى، الغدد الكظرية، الجهاز

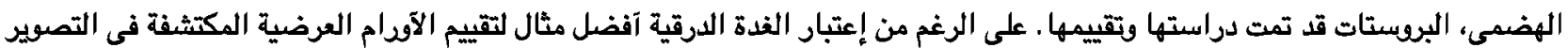

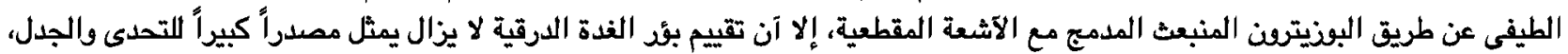

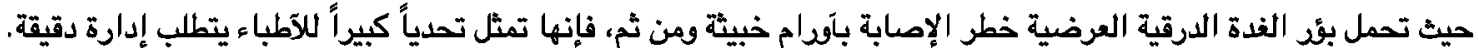

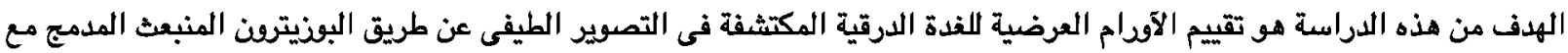
الآثعة المقطعية، وكذلك لتعديد الصد الآقصى لقيمة الإمتصاص العدية المعيارية التى يمكن آن تساعد فى عملية التشخيص.

تعد نسبة حدوث الآورام العرضية للفدة الدرقية عالية نسبياً بين المرضى الذين يقومون بالتصوير الطيفى عن طريق البوزيترون المنبعث

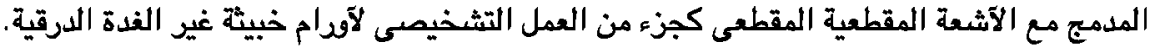

تكون قيم الإمتصاص المعيارية القصوى آعلى فى الآورام العرضية الخبيئة، ولكن مع وجود تداخل ملحوظ في القيم بين البؤد الحميدة

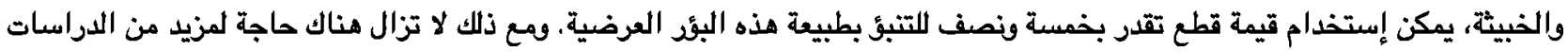

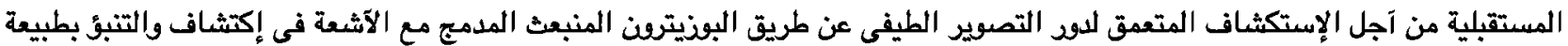
تلك البؤز الدرقية التى يتم مواجهتها بشكل متكرد.
} 